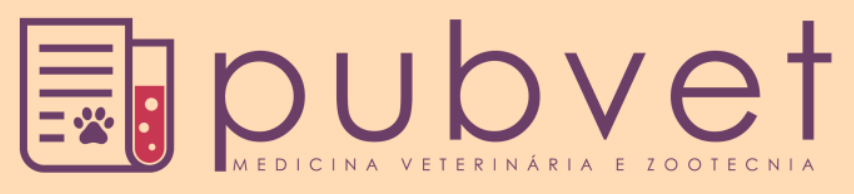

HTTP://DX.DOI.ORG/10.22256/PUBVET.V11N9.935-939

\title{
Análise multivariada da influência da ingestão de café no peso de animais
}

\section{Wederson Leandro Ferreira ${ }^{1 *}$, Fabio Vieira da Silva Junior ${ }^{2}$, Juracy Mendes Moreira ${ }^{3}$, Guido Gustavo Humada Gonzalez ${ }^{4}$, Juliano Bortolini ${ }^{5}$, Aurélio Ferreira Melo ${ }^{6}$}

\author{
${ }^{1}$ Instituto Federal Farropilha - Campus Alegrete-RS E-mail: Wederson.ferreira@iffarropilha.edu.br \\ ${ }^{2}$ Instituto Federal Farropilha - Campus Alegrete-RS E-mail: Fabio.silva@iffarropilha.edu.br \\ ${ }^{3}$ Prof mestre. Instituto Federal Goiano. Campus Rio Verde - GO E-mail:juracimendesmoreira@yahoo.com.br \\ ${ }^{4}$ Prof. Doutor. Universidad San Carlos - Asuncion-Paraguai E-mail: gustavohumad@ hotmail.com \\ ${ }^{5}$ Universidade Federal do mato Grosso - Departamento de Estatística UFMT E-mail: julianobortolini@ufmt.br \\ ${ }^{6}$ Doutorando Instituto Federal Ciência e Tecnologia Goiano Campus Rio Verde - GO E-mail: aurelioferreiramelo@hotmail.com \\ *Autor para correspondência
}

RESUMO. A metodologia de análise multivariada não impõe nenhuma restrição, assim como não há suposição sobre a estrutura da matriz de variâncias e covariâncias dos erros, tornando-se, desta forma, solução plausível para conjunto de dados longitudinais. O objetivo deste trabalho foi analisar, pelo teste de Mauchly, se a estrutura estimada desta matriz possui uma estrutura específica denominada de Huynh e Feldt, assim como utilizar a análise multivariada para avaliar se houve interação significativa, entre os períodos temporais e as seis dietas alimentares utilizadas para alimentar animais (ratos) em experimento desenvolvido no Departamento de Farmácia da Universidade Federal de Minas Gerais. Os ratos Wistar foram submetidos a seis dietas alimentares, utilizando o delineamento inteiramente casualizado com 10 repetições. Os animais foram pesados, semanalmente, durante 15 semanas, após a quarta semana do nascimento. Os resultados mostraram que os pesos dos animais são correlacionados ao longo do tempo. Ao aplicar o teste de Mauchly foi diagnosticado que a estrutura da matriz de covariâncias não atendeu a condição definida por Huynh e Feldt, o que justificou o uso da metodologia da análise multivariada, tendo essa análise indicado interação significativa entre as seis dietas alimentares e os períodos temporais avaliados. Ao proceder ao desdobramento da interação ficou evidenciado que o uso do café na dieta de ratos normonutridos acarretou um menor ganho de peso médio dos animais, quando comparado com a mesma dieta sem o uso do café.

Palavras chave: Dados longitudinais, teste de Mauchly, análise de perfil, café

\section{Multivariate analysis of influence of coffee intake on animal weight}

ABSTRACT. The multivariate analysis methodology does not impose any constraints, as there is no assumption about the structure of the variance and covariance matrix of the errors, making it a plausible solution for the longitudinal data set. The objective of this work was to analyze, by the Mauchly test, if the estimated structure of this matrix has a specific structure called Huynh and Feldt, as well as to use the multivariate analysis to evaluate if there was significant interaction between the temporal periods and the six diets Used to feed animals (rats) in an experiment developed at the Department of Pharmacy of the Federal University of Minas Gearis. Wistar rats were submitted to six feeding diets, using a completely randomized design with 10 replicates. The animals were weighed weekly for 15 weeks after the fourth week of birth. The results showed that animal weights are correlated over time. When applying the Mauchly test it was diagnosed that the structure of the covariance matrix did not meet the condition defined by Huynh and Feldt, 
which justified the use of multivariate analysis methodology, and this analysis indicated a significant interaction between the six diets and the periods Evaluated. In the unfolding of the interaction it was evidenced that the use of coffee in the diet of normonutrient rats resulted in a lower average weight gain of the animals when compared to the same diet without the use of coffee.

Keywords: longitudinal data, Mauchly's test, profile analysis, coffee

\section{Análisis multivariado da influencia de la ingesta de café sobre el peso de animales}

RESUMEN. La metodología de análisis multivariada no impone ninguna restricción, así como no hay suposición sobre la estructura de la matriz de varianzas y covariancias de los errores, convirtiéndose, de esta forma, solución plausible para el conjunto de datos longitudinales. El objetivo de este trabajo fue analizar, con la prueba de Mauchly, si la estructura estimada de esta matriz posee una estructura específica denominada de Huynh y Feldt, así como utilizar el análisis multivariado para evaluar si hubo interacción significativa entre los períodos temporales y las seis dietas alimenticias utilizadas para alimentar animales (ratas) en experimento desarrollado en el Departamento de Farmacia de la Universidad Federal de Minas Gerais. Los ratones Wistar fueron sometidos a seis dietas alimenticias, utilizando el delineamiento completamente casualizado con 10 repeticiones. Los animales fueron pesados, semanalmente, durante 15 semanas, después de la cuarta semana del nacimiento. Los resultados mostraron que los pesos de los animales se correlacionan a lo largo del tiempo. Al aplicar la prueba de Mauchly se le diagnosticó que la estructura de la matriz de covariancias no atendió a la condición definida por Huynh y Feldt, lo que justificó el uso de la metodología del análisis multivariada, habiendo este análisis indicado interacción significativa entre las seis dietas alimenticias y los períodos temporales evaluados. Al proceder al desdoblamiento de la interacción quedó evidenciado que el uso del café en la dieta de ratones normonutridos acarreó una menor ganancia de peso promedio de los animales, en comparación con la misma dieta sin el uso del café.

Palabras clave: Datos longitudinales, prueba de Mauchly, análisis de perfiles, café

\section{Introdução}

Técnicas de análises de experimentos que utilizam medidas repetidas ao longo do tempo devem considerar a estrutura de correlação inter e intra tempos. Neste trabalho foi considerado o fato relevante que, às vezes, não é estudado, ou seja, dados mensurados longitudinalmente podem ocasionar correlações as observações e consequentemente dependência dos erros. Huynh and Feldt (1970) relatam que o uso da análise de variância clássica, apenas pode ser usado em dados longitudinais se a matriz de covariância atender a uma condição específica descrita por esses autores. Entretanto, pode ser que haja uma aproximação estatística entre a matriz estimada e a desejada, sendo possível utilizar o teste de Mauchly para analisar essa aproximação.

Outra alternativa, caso à condição de $\underline{\text { Huynh }}$ and Feldt (1970) não seja atendida pelo teste de Mauchly é a utilização da análise de variância multivariada, também chamada de análise multivariada de perfil, porque não pressupõe uma estrutura específica para a matriz de covariância dos erros, apenas que os erros tenham distribuição normal multivariada.

O objetivo deste trabalho foi verificar se a matriz de covariância dos erros atende à condição de Huynh and Feldt (1970) pelo teste de Mauchly e em caso de rejeição dessa hipótese, utilizar a análise multivariada de perfil para analisar a influência do uso do café no ganho de peso médio, em gramas, de ratos.

\section{Material e Métodos}

Os dados utilizados neste foram provenientes de experimento conduzido no Departamento de Farmácia da Universidade Federal de Minas Gerais por Barbosa (2007). Foi utilizado o delineamento inteiramente casualizado, com 10 repetições, sendo os tratamentos constituídos por diferentes tipos de dietas alimentares, sendo três sem extrato de café e três com extrato de café, cuja descrição foi adaptada de Barbosa (2007), como: (N) grupo normonutrido controle - recebeu ração 
de biotério para ratos (Labina) enriquecida com ração para cães (Bonzo); (NC) grupo normonutrido café - foi alimentado com dieta do grupo normonutrido controle acrescida de extrato aquoso de café; (D60) grupo desnutrido controle foi alimentado com dieta correspondente a $60 \%$ da ração consumida pelo grupo normonutrido controle; (D60C) grupo desnutrido café - recebeu dieta do grupo desnutrido controle, acrescida de extrato aquoso de café; (D60R) grupo desnutrido com condição alimentar reconstituída controle foi alimentado com dieta correspondente a $60 \%$ da ração consumida pelo grupo normonutrido controle e, após 60 dias, foi alimentado ad libitum e (D60RC) grupo desnutrido com condição alimentar reconstituída café - recebeu dieta do grupo desnutrido acrescida de extrato aquoso de café e, após 60 dias, alimentado ad libitum.

Vieira et al. (2007) citam que uma condição suficiente para que o teste $\mathrm{F}$ da análise de variância usual, quando se utilizam esquemas de parcelas subdivididas no tempo seja exato, em nível de subparcela necessariamente, é que a matriz de covariâncias dos erros $\Sigma$ tenha uma forma uniforme. Huynh and Feldt (1970) descreveram uma condição necessária e suficiente mais geral da forma de $\Sigma$, que também garante que os testes $\mathrm{F}$ referentes à subparcela sejam válidos. Tal condição, simbolizada por (H-F) de Huynh-Feldt, especifica que os elementos da matriz de covariâncias sejam expressos, para um $\lambda>0$, de tal forma que $\operatorname{var}\left(x_{i}-x_{j}\right)=2 \lambda$, para $i \neq$ $j$, em que $\lambda$ é a diferença entre a média das variâncias e a média das covariâncias.

Pode ocorrer que a matriz de covariância dos erros não atenta à condição numérica imposta pela condição (H-F), porém, pode ser que haja uma aproximação estatística. Sendo assim, Huynh and Feldt (1970) propuseram um teste de esfericidade a partir do teste de Mauchly (1940) para verificar se a matriz de covariâncias $\sum^{*}$ satisfaz à condição de esfericidade. Os mesmos autores provaram que, se $\sum$ atender à condição $\mathrm{H}-\mathrm{F}$, então, $\Sigma^{*}$ pode ser escrita como: $\sum^{*}=C \sum C^{\prime}=\lambda I_{t-1}$, em que $C_{(t-1) \times t}$ é uma matriz de dimensões $(t-1) \times t$, que pode ser construída como uma matriz que define os coeficientes de $t-1$ contrastes ortonormais entre as $t$ medidas de tempo. Para verificar a igualdade, Huynh and Feldt (1970) descreveram um teste a partir do teste de esfericidade de Mauchly.

Para a sua aplicação, como foi descrito por Huynh and Feldt (1970) é utilizada uma matriz de contrastes ortonormais $C$ que consiste em transformar a matriz de covariância $\sum$ original para uma forma ortonormalizada $\sum^{*}$, cuja esfericidade será aferida pelo teste e, caso $\sum^{*}$ seja tida como esférica, a matriz original $\sum$, atenderá à condição H-F e implicará na garantia que os testes F da subparcela, no caso da parcela subdividida no tempo possuirá distribuição $\mathrm{F}$ exata. Caso contrário, alternativas de análises, como é o caso da análise de variância multivariada deve ser preferida, segundo Ferreira and Moraies (2013). A seguir, a descrição do teste.

Sob a hipótese nula, $H_{o}: \sum^{*}=C \sum C^{\prime}=$ $\lambda I_{t-1}$ que é a hipótese de que a matriz de covariância satisfaz à condição de esfericidade, a estatística W do teste Mauchly (1940) poderá ser utilizada para testar esta hipótese de independência e homocedasticidade de uma variável aleatória normal multivariada. Se $S$ é a matriz de covariância amostral para o erro experimental (intraindivíduo) da subparcela e $p=$ $t-1$, o número de contrastes ortogonais, então, $o$ valor de $\mathrm{W}$ é dado.

$$
W=\frac{\left|C S C^{\prime}\right|}{\left[\operatorname{tr} C S C^{\prime} / p\right]^{p}}
$$

sendo que o valor de $\mathrm{W}$ não depende da escolha particular para esta matriz de transformação C. Se $\sum$ é estimada com $n_{1}$ graus de liberdade e se considerar $f=[p(p+1) / 2]-1$ e $d=1-$ $\left(2 p^{2}+p+2\right) / 6 p n_{1}$, então se $-n_{1} d \ln W>$ $\chi_{\alpha, f}^{2}$ rejeita-se a hipótese nula a $\alpha \%$ de significância.

Em sua forma matricial, a representação do modelo multivariado é:

$$
Y=X B+E,
$$

sendo $Y$ a matriz dos dados observados de $t$ variáveis respostas para as $n$ unidades experimentais de dimensões $(n \times t) ; X$ é a matriz de delineamento conhecida, de dimensões $g k \times$ $(g+1)$, para o delineamento inteiramente casualizado com $g$ tratamentos e $k$ repetições; $B$ é a matriz dos parâmetros, de dimensões $(g+1) \times$ $t$ e $E$ é a matriz dos erros experimentais, de dimensões $n \times t$. Com as suposições que $E \sim N_{t}(\Phi, \Sigma)$, é demonstrável que $Y$ $\sim N_{t}(X B, \Sigma)$, sendo a matriz de covariância $\Sigma$ não estruturada, com $t(t+1) / 2$ parâmetros e $\Phi$ um vetor de zeros. Para situações como esta, podem-se avaliar três hipóteses de interesse, 
representadas por: $\mathrm{H}_{01}$ : não existência de efeito do fator tempo (semanas) (perfis horizontais); $\mathrm{H}_{02}$ : não existência de efeito entre tratamentos e tempo (semanas) (perfis paralelos); $\mathrm{H}_{03}$ : não existência de efeito do fator tratamento (semanas) (perfis coincidentes).

Segundo Ferreira (2011), estatísticas de testes multivariados são obtidas por meio dos princípios da união-intersecção de Roy e da razão de verossimilhança de Wilks. As principais estatísticas são:

1) Lambda de Wilks

$$
\Lambda=\prod_{i=1}^{s} \frac{1}{1+\lambda_{i}}
$$

2) Raiz máxima de Roy

$$
\theta_{\mathrm{s}}=\max \left(\lambda_{i} /\left(1+\lambda_{i}\right)\right)
$$

3) Traço de Hotelling-Lawley

$$
T=\sum_{i=1}^{s} \lambda_{i}
$$

4) Traço de Pillai

$$
P=\sum_{i=1}^{s} \frac{\lambda_{i}}{1+\lambda_{i}} .
$$

Nas quais $\lambda_{i}$ é a i-ésima raiz latente de $H E^{-1}$ e $S$ é o número das raízes latentes não nulas, sendo $S=\min (t-1 ; g-1)$. As distribuições exatas dessas estatísticas dependem, sob-hipótese nula, dos parâmetros $m_{1}=(|(t-1)-(g-1)|-1) /$ 2 e $m_{2}=(n-g-(t-1)-1) / 2$. Detalhes do cálculo de $H$ e $E$, podem ser encontrados em Ferreira (2011).

Para a comparação das médias das dietas nos tempos (semanas) individuais, usou-se o teste de Tukey ao nível de 5\% de probabilidade.

\section{Resultados e Discussões}

Procedendo-se a análise do teste de Mauchly (1940), via software SAS, constata-se que o teste rejeitou a hipótese de esfericidade de $\Sigma^{*}$, o que implica que a matriz de covariâncias do erro experimental $\sum$ não atende à condição proposta por Huynh and Feldt (1970) (Tabela 1), implicando que a análise multivariada de perfil é uma alternativa mais plausível para analisar o conjunto de dados, pois, pelo teste, $\sum$ não possui uma estrutura de correlação homocedastica.
Tabela 1. Resultado do teste de esfericidade de Mauchly.

\begin{tabular}{lccc}
\hline $\begin{array}{l}\text { Graus de } \\
\text { liberdade } f\end{array}$ & $\begin{array}{c}\text { Critério de } \\
\text { Mauchly ( W) }\end{array}$ & $\begin{array}{c}\text { Estatística de Qui Valor - } \\
\text {-quadrado } \chi^{2}\end{array}$ & $\begin{array}{c}\text { p } \\
104\end{array}$ \\
\hline
\end{tabular}

Ao usar o proc GLM, do SAS foram impressos os quatro testes multivariados. Pelos testes multivariados (Tabela 2), testou-se a hipótese nula de perfis horizontais, ou seja, que testa o efeito do fator tempo. Para a fonte de variação que foi testada, foi obtida uma matriz $H$ de soma de quadrados e produtos, além da matriz $E$ de soma de quadrados e produtos referentes aos erros do modelo. Na Tabela 2, também foram impressos os valores $S, m_{1}$ e $m_{2}$, utilizados para obter a aproximação com a estatística $F$.

Tabela 2. Resultado da análise multivariada da variável peso, em gramas, dos animais, considerando o delineamento inteiramente casualizado e testando o efeito nulo do fator tempo.

\begin{tabular}{lrcccc}
\hline \multicolumn{5}{c}{$S=1, m_{1}=0.5$ e $m_{2}=1$} \\
\hline Estatística & Valor & $\mathrm{F}$ & $\begin{array}{c}\text { G.L. } \\
\text { Num }\end{array}$ & $\begin{array}{c}\text { G.L. } \\
\text { Den }^{2} .\end{array}$ & $\begin{array}{c}\text { Valor }- \\
\mathrm{p}\end{array}$ \\
\hline $\begin{array}{l}\text { Lambda de } \\
\text { Wilks }\end{array}$ & 0,0025 & 1138,83 & 14 & 41 & $<0,001$ \\
$\begin{array}{l}\text { Traço de Pillai } \\
\text { Traço de H. }\end{array}$ & 0,9974 & 1138,83 & 14 & 41 & $<0,001$ \\
$\begin{array}{l}\text { Lawley } \\
\text { Raiz máxima de }\end{array}$ & 388,8680 & 1138,83 & 14 & 41 & $<0,001$ \\
Roy & 388,8680 & 1138,83 & 14 & 41 & $<0,001$ \\
\hline
\end{tabular}

Pelos testes multivariados (Tabela 3), testou-se a hipótese nula de perfis paralelos que testa a interação entre dietas $\times$ tempo. Na Tabela 3 primeiramente foram utilizadas a matriz $H$ da soma de quadrados e produtos para a interação de semanas $\times$ dietas e a matriz $E$ dos erros.

Tabela 3. Resultado da análise multivariada da variável peso,

\begin{tabular}{|c|c|c|c|c|c|}
\hline \multicolumn{6}{|c|}{$\mathrm{S}=2, \mathrm{~m}_{1}=0$ e $\mathrm{m}_{2}=1$} \\
\hline Estatística & Valor & $\mathrm{F}$ & $\begin{array}{l}\text { G.L. } \\
\text { Num1. }\end{array}$ & $\begin{array}{l}\text { G.L. } \\
\text { Den2. }\end{array}$ & $\begin{array}{c}\text { Valor - } \\
\mathrm{p}\end{array}$ \\
\hline $\begin{array}{l}\text { Lambda de } \\
\text { Wilks }\end{array}$ & 0,0002 & 12,84 & 70 & 199,29 & $<0,001$ \\
\hline Traço de Pillai & 3,3496 & 6,52 & 70 & 225,00 & $<0,001$ \\
\hline $\begin{array}{l}\text { Traço de H.- } \\
\text { Lawley }\end{array}$ & 45,8301 & 25,84 & 70 & 129,07 & $<0,001$ \\
\hline $\begin{array}{l}\text { Raiz máxima de } \\
\text { Roy }\end{array}$ & 30,9503 & 99,48 & 14 & 45 & $<0,001$ \\
\hline
\end{tabular}
em gramas, dos animais, considerando o delineamento inteiramente casualizado e testando efeito nulo da interação de dietas $\times$ tempo. 
Observa-se, na Tabela 2, que a hipótese de perfis horizontais que testa o efeito do fator tempo e a hipótese de perfis paralelos (Tabela 3 ), que testa o efeito da interação de dietas $\times$ tempo, foram, ambas rejeitadas por todos os testes, a $1 \%$ de probabilidade. Isso indica, pela hipótese de paralelismo, que pelo menos uma combinação de dietas e tempos interage de forma dependente.

Após confirmação da significância da interação tempo $\mathrm{x}$ dietas, passa-se ao desdobramento de interesse (Tabela 4). É nítido perceber durante a evolução do tempo que o uso da dieta NC tendeu a diminuir o ganho médio de peso dos animais, quando comparado com a dieta $\mathrm{N}$ pelo teste de Tukey a 5\% de probabilidade. Portanto, como estas dietas diferem apenas pelo uso do café é razoável afirmar, neste caso, que o café diminui o ganho médio de peso dos animais ao usar a dieta NC. Ao comparar as demais dietas, não houve diferenças significativas quanto ao uso, ou não, do café.

Tabela 4. Peso médio dos animais considerando com o uso de seis dietas alimentares durante a $3^{\mathrm{a}}, 6^{\mathrm{a}}, 9^{\mathrm{a}}, 12^{\mathrm{a}}$ e $15^{\mathrm{a}}$ semanas em que os animais foram avaliados.

\begin{tabular}{|c|c|c|c|c|c|}
\hline Dietas & $\begin{array}{c}3^{\mathrm{a}} \\
\text { semana } \\
\end{array}$ & $\begin{array}{c}6^{\mathrm{a}} \\
\text { semana } \\
\end{array}$ & $\begin{array}{c}9^{\mathrm{a}} \\
\text { semana } \\
\end{array}$ & $\begin{array}{c}12^{\mathrm{a}} \\
\text { semana } \\
\end{array}$ & $\begin{array}{c}15^{\mathrm{a}} \\
\text { semana }\end{array}$ \\
\hline $\mathrm{N}$ & $161,1 \mathrm{~A}$ & $325,2 \mathrm{~A}$ & $415,5 \mathrm{~A}$ & $465,0 \mathrm{~A}$ & $479,3 \mathrm{~A}$ \\
\hline $\mathrm{NC}$ & $141,9 \mathrm{~A}$ & 283,9 B & 366,5 B & $402,4 \mathrm{~B}$ & 429,2 B \\
\hline D60R & 83,2 B & $170,3 \mathrm{D}$ & $287,6 \mathrm{C}$ & $357,8 \mathrm{C}$ & $393,9 \mathrm{C}$ \\
\hline D60RC & 79,2 B & $155,1 \mathrm{D}$ & $283,1 \mathrm{C}$ & $346,4 \mathrm{C}$ & $380,0 \mathrm{C}$ \\
\hline D60 & 91,4 B & $189,1 \mathrm{C}$ & $241,0 \mathrm{D}$ & $271,6 \mathrm{D}$ & $297,9 \mathrm{D}$ \\
\hline D60C & $80,8 \mathrm{~B}$ & $170,2 \mathrm{D}$ & $222,9 \mathrm{D}$ & $257,5 \mathrm{D}$ & $288,2 \mathrm{D}$ \\
\hline
\end{tabular}

Médias seguidas da mesma letra não diferem, entre si, pela coluna, pelo teste de Tukey a $5 \%$ de probabilidade.

\section{Conclusões}

O teste de Mauchly indicou que os dados avaliados em uma mesma unidade experimetal foram correlacionados, fato que justificou o uso da metodologia estatística de análise multivariada.

Houve concordância entre os quatro testes multivariados ao analisar a interação das dietas alimentares e períodos (semanas) avaliados.
$\mathrm{O}$ uso do café na dieta $\mathrm{NC}$ tendeu, em média, influenciar um menor ganho de peso médio dos animais, quando comparado com a mesma dieta, entretanto sem o uso do café.

\section{Referências Bibliográficas}

Barbosa, W. M. 2007. Efeito da ingestão de café sobre a liberação de glutamato sinaptossomal, estimulda por toxina escorpiônica, em ratos. Universidade Federal de Minas Gerais, Belo Horizonte.

Ferreira, D. F. 2011. SISVAR: A Computer Statistical Analysis System. Ciência e Agrotecnologia, 35, 1039-1042.

Ferreira, W. L. \& Moraies, A. R. 2013. Análise da influência do café no ganho de peso de animais (ratos) por meio de modelo linear misto. Revista Brasileira de Biometria, 31, 485-500.

Huynh, H. \& Feldt, L. S. 1970. Conditions under which mean square ratios in repeated measurements designs have exact Fdistributions. Journal of the American Statistical Association, 65, 1582-1589.

Mauchly, J. W. 1940. Significance test for sphericity of a normal n-variate distribution. The Annals of Mathematical Statistics, 11, 204-209.

Vieira, F. T. P. A., Silva, J. A. A., Ferreira, R. L. C., Cruz, M. A. O. M. \& Ferraz, I. 2007. Uma abordagem multivariada em experimentos Silvipastoral com Leucaena leucocephala (Lam.) de Wit no Agreste de Pernambuco. Ciência Florestal, 17, 333-342.

\section{Article History:}

Received 24 April 2017

Accepted 16 May 2017

Available online 8 August 2017

License information: This is an open-access article distributed under the terms of the Creative Commons Attribution License 4.0, which permits unrestricted use, distribution, and reproduction in any medium, provided the original work is properly cited. 